Nota de investigación

\title{
Actitud del productor agrícola ante la transferencia de tecnología en la región centro de Sinaloa
}

\author{
Guadalupe Alfonso López \\ Urquídez, Moises Gilberto Yañez Juárez \\ Teresa de Jesús Velázquez Alcaraz \\ Felipe Ayala Tafoya \\ Carlos Alfonso López Orona ${ }^{\S}$
}

Facultad de Agronomía-Universidad Autónoma de Sinaloa. Carretera Culiacán-Eldorado km 17.5, Culiacán, Sinaloa, México. (alfonsolopezurquidez@uas.edu.mx; moisesyj@uas.edu.mx; teresadejesus_ v@yahoo.com.mx; tafoya@uas.edu.mx).

${ }^{\S}$ Autor para correspondencia: clopezorona@uas.edu.mx.

\section{Resumen}

Se evaluó la actitud del productor agrícola de la zona centro de Sinaloa (México) ante la transferencia de tecnología, para generar estrategias que permitan facilitar la innovación tecnológica en la agricultura. Se empleó un cuestionario a los productores, quienes fueron seleccionados mediante el método muestreo por lotes del control de calidad (LQAS), conformándose cinco lotes, aplicando 19 cuestionarios en cada uno. Se encontró que la mayoría de los productores utiliza la nueva tecnología una vez aplicada por otros productores que tuvieron resultados positivos, recurren principalmente al proveedor para resolver un problema técnico, al agricultor no le significan las cosas que no lo afectan en lo inmediato, pero cuando considera que sus intereses son afectados de manera directa e inmediata, su actitud es diferente. No le importan las reuniones sobre transferencia de tecnología porque no conoce la utilidad de estas, la conducta del agricultor es influida por la opinión de los familiares y los proveedores, la principal fuente de información sobre tecnología agrícola es el proveedor de insumos, aunque también se informan por medio del amigo o familiar; así como de demostraciones sobre novedades tecnológicas y exposiciones agropecuarias.

Palabras clave: conducta, familia, innovación, proveedor.

Recibido: agosto de 2019

Aceptado: septiembre de 2019 
En el presente trabajo de investigación, la transferencia de tecnología se estudia en el contexto de la problemática del medio rural que ha intentado resolver el gobierno de México a lo largo de varios años y a partir de la cual se formula la Ley de Desarrollo Rural Sustentable (LDRS), que en su artículo 32 señala que las acciones y programas que se establezcan para impulsar el desarrollo rural se orientarán a incrementar la productividad y la competitividad en ámbito rural, a fin de fortalecer el empleo y elevar el ingreso de los productores.

Para ello en el mismo artículo en la fracción I se indica que se propiciará: el impulso a la investigación y desarrollo tecnológico, la apropiación de tecnología y su validación, así como transferencia de tecnología a los productores (Cámara de Diputados del H. Congreso de la Unión, 2012). El problema de la improductividad agrícola aplica a Sinaloa, donde en 2013 se sembraron 838278.58 ha en la zona de riego y 369895.59 ha en el área de temporal. Entre ambos sistemas de producción existen diferencias en cuanto a rendimiento, por ejemplo, en maíz bajo sistema de riego, en 2013 se obtuvo un promedio de $10.13 \mathrm{tha}^{-1} \mathrm{y}$ en temporal fue de $1.04 \mathrm{t} \mathrm{ha}^{-1}$. (SIAP, 2013).

Asimismo, dentro de las zonas de riego también hay diferencias en productividad, a pesar de que se cuenta con la tecnología para incrementar la producción y la calidad por unidad de superficie, pues hay técnicas de producción que son más amigables con el medio ambiente y reducen los costos de producción, las cuales no están generalizadas entre los agricultores, por lo que su aplicación permitiría mayor rentabilidad y un manejo sostenible de los recursos naturales.

La mayor parte de los productores no adopta inmediatamente las nuevas propuestas tecnológicas generadas en los centros de investigación. Por ejemplo, Fundación Produce Sinaloa ha recomendado sembrar la semilla de maíz a doble hilera o una separación entre surcos de $50 \mathrm{~cm}$ para obtener mayores rendimientos con respecto a la separación tradicional $(75 \mathrm{u} 80 \mathrm{~cm})$, pero los productores aun no la han adoptado, ya que la transferencia tecnológica implica un traslado planificado de la información y técnicas de cómo llevar a cabo las actividades de adopción, asimilación y aprendizaje de habilidades y conocimientos (Herrera, 2006), por ello es necesario tener conocimientos sobre la actitud del productor.

Hay otros factores que limitan la correcta aplicación de la nueva tecnología (Damián et al. (2007). En el proceso de difusión de la tecnología, el productor puede pasar por diferentes etapas, en las cuales se ven reflejados los distintos elementos actitudinales, tales como valor de importancia, conducta, la intención y los conocimientos (Corro, 2007). Hay productores agrícolas que rechazan la propuesta de innovación tecnológica, aunque algunos adoptan la tecnología inmediatamente.

Conocer la actitud del productor agrícola ante la propuesta de nuevas formas producción agrícola es muy importante para generar estrategias que sean eficientes en el proceso de innovación tecnológica, puesto que los procesos de transferencia de tecnología requieren promover que más productores pongan a prueba todos o al menos algunos de los componentes tecnológicos para lograr la transferencia de los conocimientos (Hernández et al., 2008). El objetivo del trabajo fue determinar la actitud del productor agrícola ante las nuevas propuestas de innovación tecnológica, para generar estrategias que permitan facilitar la innovación tecnológica en la agricultura.

El estudio se realizó en la zona centro del estado de Sinaloa, ubicado entre los $22^{\circ} 20^{\prime}$ y $27^{\circ} 07^{\prime}$ de latitud norte y entre los $105^{\circ} 22^{\prime}$ y $109^{\circ} 30^{\prime}$ longitud oeste y una altitud que varía de 0 hasta 2520 m (INEGI y Gobierno del estado de Sinaloa, 2012). 
Se eligió a los productores por el método muestreo por lotes del control de calidad (LQAS). (Davis et al., 2009; FAO, SAGARPA, 2010). Se conformaron cinco lotes con base en los centros de apoyo al desarrollo rural (CADER), que se encuentran en el centro del estado. Se fusionó al CADER Culiacán Altos con el CADER Badiraguato, ya que en ambos se desarrolla agricultura de temporal. También, se fusionó el CADER Navolato con El Tamarindo, porque en ellos hay una interacción geográfica y se desarrolla agricultura de riego. Los cinco lotes resultantes son: Culiacán Valle (1); Eldorado (2); Angostura (3); Navolato-El Tamarindo (4); y Culiacán Altos-Badiraguato (5).

El tamaño de muestra se calculó con base en tablas del LQAS, con un nivel de confianza de 95\%, obteniéndose un tamaño de muestra de 19 productores por lote, para un tamaño de muestra de 95. Posteriormente se realizó un muestreo aleatorio simple para seleccionar al productor a encuestar. Para ello se estimó el intervalo de muestreo dividiendo el número de productores de cada lote entre 19 como lo indica el modelo. Además, el listado de beneficiarios por lote fue ordenado alfabéticamente y se le asignó un número en orden ascendente.

Posteriormente, se calcularon los 19 números de manera sistemática a partir del primer valor seleccionado aleatoriamente en la hoja de cálculo Excel, indicando que el valor mínimo es 1, mientras que el valor máximo fue el intervalo de muestreo. Previendo que algunos productores no respondieran a la entrevista, se realizó un listado de reemplazos equivalente a $20 \%$ del total de la muestra por lote, con base en el procedimiento descrito anteriormente.

Se utilizaron encuestas con preguntas abiertas y cerradas, con tres secciones: a) Identificación del Productor, en el que se incluyeron preguntas como el nivel de escolaridad, sexo, edad; b) difusión de la transferencia de tecnología; y c) actitudes, el cual se subdividió en valor de importancia, intención, acción, control y normatividad.

Las encuestas se aplicaron durante los meses de febrero y marzo de 2012. Para ello el cuestionario fue examinado mediante una prueba piloto con diferentes productores para detectar problemas de redacción que condujeran a la mala interpretación de la pregunta, así como para calcular la duración de la entrevista, la secuencia lógica de las preguntas, ver si las escalas de medición eran suficientes, la codificación y procesamiento de la información. Para determinar la fiabilidad del cuestionario, se aplicó el coeficiente de confiabilidad Alfa de Conbrach que permitió evaluar la consistencia de los resultados obtenidos (Cuadro 1). Este coeficiente fue medido mediante la varianza de los ítems y la varianza del puntaje total.

Cuadro 1. Coeficientes de fiabilidad de los cuestionarios utilizados.

\begin{tabular}{lcccc}
\hline Variable de actitud & $\begin{array}{c}\mathrm{S}^{2} \text { la } \\
\text { población }\end{array}$ & $\begin{array}{c}\mathrm{S}^{2} \text { de la suma de } \\
\text { items }\end{array}$ & $\begin{array}{c}\text { Núm. de } \\
\text { ítems }\end{array}$ & $\begin{array}{c}\text { Alfa de } \\
\text { Cronbach }\end{array}$ \\
\hline $\begin{array}{l}\text { Valor de importancia } \\
\text { Normatividad }\end{array}$ & 22.9 & 126.22 & 18 & 0.87 \\
subjetiva & 28.74 & 94.71 & 21 & 0.73 \\
Percepción de control & 14.05 & 55.84 & 10 & 0.83 \\
Intensión & 9.86 & 36.01 & 7 & 0.85 \\
Acciones & 14.05 & 55.84 & 10 & 0.83 \\
\hline
\end{tabular}


Con respecto a la búsqueda de información tecnológica sobre agricultura se encontró que 3\% de ellos nunca lo hace, $18 \%$ casi nunca no realiza, $37 \%$ a veces, $23 \%$ casi siempre busca información y solo $18 \%$ de los productores siempre está tratando de obtener información que le permita mejorar su proceso productivo. En complemento con lo anterior el productor agrícola considera que cuando se le presenta un problema siempre $(40 \%)$ o casi siempre $(35 \%)$ se basa en su experiencia como productor para resolverlo.

Se encontró que $2.04 \%$ de los productores aplica la nueva tecnología al participar en la validación de tecnología junto con los investigadores, el $18.37 \%$ aplica la nueva tecnología en cuanto tiene conocimiento de ella, $6.12 \%$ lo realiza muy rara vez, mientras que $37.76 \%$ aplica la tecnología una vez que vio que la aplicaron otros productores, $33.67 \%$ lo hace hasta que observa resultados positivos en los demás productores y solo $2.04 \%$ nunca aplica la nueva tecnología. Esto coincide con la curva de difusión de la tecnología de Rogers (2003). Sin embargo, aunque la curva de difusión se mantenga, se puede incidir en los productores para que el tiempo de asimilación disminuya entre cada grupo de agricultores, difundiendo los resultados obtenidos por los primeros productores adoptadores de la nueva tecnología.

Se observó que $43 \%$ de los productores cuenta con el servicio del internet en sus hogares; sin embargo, solo 7\% de los productores la utiliza. Al respecto, González (2012) indica que existen los influenciadores de opinión, que son aquellos que cuando encuentran en internet información que les gusta y les llama la atención lo comentan con sus conocidos. Si el productor no accede a este servicio, sus familiares sí lo hacen, entonces es importante recurrir a los influenciadores para transferir tecnología.

Se encontró que $27 \%$ de los productores siempre recurre al proveedor de insumos para solicitar asesoría ante un problema relacionado con la producción agrícola y $37 \%$ casi siempre recurre al mismo. Lo anterior, refleja la importancia que para el productor tiene el proveedor de insumos. Por el contrario, $66 \%$ de los productores nunca o casi nunca recurre a una institución educativa o de investigación para solicitar asesoría. Una de las razones de ello tal vez sea que no existen mecanismos de vinculación adecuados entre las instituciones y los productores, además de que el investigador da a conocer sus conocimientos a través de revistas y eventos científicas, a los cuales el agricultor no tiene acceso.

Se observó que el agricultor le da más importancia a las cosas que lo afectan en lo inmediato, pues $57 \%$ de los productores le da demasiada importancia a la aplicación de nitrógeno a los cultivos y $31 \%$ le da mucha importancia, por ello siempre realiza las aplicaciones de este nutriente. También le da mucha importancia a la calidad de la semilla, pues sabe que en las características de ella son determinantes en el rendimiento del cultivo. Lo anterior, significa que la conducta del productor será influida de acuerdo con la importancia que le da a determinado fenómeno (Ajzen y Fishbein, 1980).

En contraparte se observó que solo para $11 \%$ de los productores tienen importancia los eventos de transferencia de tecnología. Mientras que $28 \%$ las reuniones de transferencia tienen mediana importancia. Una de las razones de estos resultados es que ellos no conocen la utilidad de estos eventos. El productor norma su conducta por la opinión de los familiares. Un amplio porcentaje lo hace para satisfacer la opinión de la familia, pues $38 \%$ indica que casi siempre y $18 \%$ señala que siempre que hace adecuaciones en su forma de producir es para complacer a los familiares. 
Al respecto, Bravo et al. (2006) señalan que la familia influye para que el adulto joven adquiera determinado producto. Muchos de los productores agrícolas han tenido, tienen o tendrán a sus hijos estudiando en una escuela de agricultura. Este puede ser el medio para que la tecnología sea transferida a los agricultores. Otro personaje que influye mucho en la conducta del productor es el proveedor de insumos. El agricultor considera que el proveedor desea que se aplique la nueva tecnología. El 54\% considera que el proveedor casi siempre desea que aplique la nueva tecnología, mientras que $34 \%$ indica que todo el tiempo desea que esté innovando.

El 52\% de los productores opina que a las autoridades de gobierno no les interesa que en el campo se aplique nueva tecnología, mientras que el $30 \%$ opina que casi nunca le interesa que el productor aplique nueva tecnología. En el corto tiempo es muy complicado cambiar la opinión del productor. Ante ello el gobierno puede utilizar otras instancias, tales como a las instituciones de investigación y a los propios proveedores. El $28 \%$ de los productores cree que la familia siempre desea que aplique la nueva tecnología, mientras que $35 \%$ percibe que la familia sí quiere que cambie su forma de trabajar la tierra.

Los productores consideran que la duración de la reunión no debe ser mayor a las 2 h, que el lugar para que se realicen las reuniones sean en el campo o en el espacio en donde regularmente se reúnen los productores. Es importante realizar reuniones de trabajo en el espacio adecuado para ello. Por eso se debe ir previamente al lugar a donde el productor se reúne cotidianamente, para posteriormente, llevarlos a otros espacios mediante la motivación (Salinas et al., 2006). La gran mayoría de los productores considera que las reuniones sobre transferencia de tecnología fueran los sábados o los domingos a las 10:00 h.

\section{Conclusiones}

Para hacer más eficiente la transferencia de tecnología, hay que apoyarse en los productores cuyos objetivos se fusionan con los de su grupo de pertenencia. Ellos se encargarán de transmitir sus conocimientos al resto de personas de la colectividad. Hay que buscar la colaboración con las empresas proveedoras de insumos para que sus técnicos asistan a talleres en donde se transmita la tecnología y sea validada por los diferentes centros de investigación de la entidad, para que ellos sean el vehículo a través del cual la tecnología sea transferida hacia los productores. Se debe motivar al productor, primero para que asista a la reunión o a la demostración de campo, después, tener la capacidad para motivarlo a que aplique la nueva tecnología propuesta, dándole a conocer los beneficios que puede obtener en ellas.

\section{Literatura citada}

Ajzen, I. y Fishbein, M. 1980. Understanding attitudes and predicting social behavior. Ed. PrenticeHall. Englewood Cliffs, NJ. 278 p.

Bravo, G. R.; Fraj, E. y Martínez, S. E. 2006. Factores determinantes de las influencias familiares en el comportamiento de compra. Un enfoque desde la perspectiva del joven adulto. Investigaciones Europeas de la Dirección y Economía de la Empresa. 12(2):91-105.

C de D. 2012. Cámara de Diputados del H. Congreso de la Unión. Ley de Desarrollo Rural Sustentable. DOF. México, DF. 
Corro, M. M. D. 2007. Factores que determinan la adopción de tecnología en el área de reproducción de ganado bovino. www.fmvz.unam.mx/fmvz/departamentos/.../ bovinotecnia/BtRgz00g030.pdf.

Damián, H. M. A.; Ramírez, V. B.; Parra, I. F.; Paredes, S. J. A.; Muñoz, A. G.; Cruz, L. A. y López, O. J. F. 2007. Apropiación de tecnología por productores de maíz en el estado de Tlaxcala, México. Agric. Téc. Méx. 33(2):163-173.

Davis, R.; Luna, J.; Rodríguez, L. A. y Sarriot, E. 2009. El manual de encuestas rápidas de hogares: cómo obtener información confiable sobre salud a nivel local. ICF Macro: Calverton, M. D. y Public Health Institute: Oakland, CA. 174 p.

FAO-SAGARPA. 2010. Instrumentos metodológicos para el monitoreo 2009 y evaluación de desempeño. Food and Agriculture Organization (FAO)-Secretaría de Agricultura, Ganadería, Desarrollo Rural, Pesca y Alimentación (SAGARPA). 89 p.

González, J. 2012. Transferencia de tecnología 2. Conectar hoy con empresas desde universidades y centros tecnológicos. Ed. The Transfer Institute. Alicante, España. 78 p.

Hernández, C. E.; Martínez, D. P.; Gallardo, L. F. y Villanueva, J. J. A. 2008. Aceptación de nueva tecnología por productores ejidales para el manejo integrado del cultivo de papayo. Trop. Subtrop. Agroecosys. 8(3):279-288.

Herrera, T. F. 2006. Innovaciones tecnológicas en la agricultura empresarial mexicana. Una aproximación teórica. Gaceta Laboral. 12(1):91-117.

INEGI. 2012. Anuario estadístico del estado de Sinaloa 2008. Instituto Nacional de Estadística y Geografía y Gobierno del estado de Sinaloa. $442 \mathrm{p}$.

Rogers, E. M. 2003. Diffusion of innovations. $5^{\text {a }}$ (Ed.). New York: Free Press Rogers, C. R. Grupos de encuentro. 2004. Amorrortu. Buenos Aires. 192 p.

Salinas, A. B.; Huerta, M. G.; Porras, L. H.; Amador, S. E. y Ramos, J. M. 2006. Uso significativo de la tecnología en la educación de adultos en el medio rural: resultados de la aplicación piloto de un modelo. Rev. Mex. Invest. Ed. 11(28):31-60. 